\title{
Definition and some Properties of Information Entropy
}

\author{
Bo Zhang \\ Shinshu University \\ Nagano, Japan
}

\author{
Yatsuka Nakamura \\ Shinshu University \\ Nagano, Japan
}

Summary. In this article we mainly define the information entropy [3], [11] and prove some its basic properties. First, we discuss some properties on four kinds of transformation functions between vector and matrix. The transformation functions are LineVec2Mx, ColVec2Mx, Vec2DiagMx and Mx2FinS. $\mathrm{Mx} 2 \mathrm{FinS}$ is a horizontal concatenation operator for a given matrix, treating rows of the given matrix as finite sequences, yielding a new finite sequence by horizontally joining each row of the given matrix in order to index. Then we define each concept of information entropy for a probability sequence and two kinds of probability matrices, joint and conditional, that are defined in article [25]. Further, we discuss some properties of information entropy including Shannon's lemma, maximum property, additivity and super-additivity properties.

MML identifier: ENTROPY1, version: 7.8.05 4.84.971

The papers [21], [23], [1], [20], [24], [6], [14], [8], [4], [22], [17], [7], [9], [2], [5], [15], [16], [12], [10], [13], [18], [25], and [19] provide the terminology and notation for this paper.

\section{Preliminaries}

For simplicity, we use the following convention: $D$ denotes a non empty set, $i, j, k, l$ denote elements of $\mathbb{N}, n$ denotes a natural number, $a, b, c, r, r_{1}, r_{2}$ denote real numbers, $p, q$ denote finite sequences of elements of $\mathbb{R}$, and $M_{1}, M_{2}$ denote matrices over $\mathbb{R}$.

Next we state several propositions:

(1) If $k \neq 0$ and $i<l$ and $l \leq j$ and $k \mid l$, then $i \div k<j \div k$. 
(2) If $r>0$, then $\left(\log _{-}(e)\right)(r) \leq r-1$ and $r=1$ iff $\left(\log _{-}(e)\right)(r)=r-1$ and $r \neq 1$ iff $\left(\log _{-}(e)\right)(r)<r-1$.

(3) If $r>0$, then $\log _{e} r \leq r-1$ and $r=1$ iff $\log _{e} r=r-1$ and $r \neq 1$ iff $\log _{e} r<r-1$.

(4) If $a>1$ and $b>1$, then $\log _{a} b>0$.

(5) If $a>0$ and $a \neq 1$ and $b>0$, then $-\log _{a} b=\log _{a}\left(\frac{1}{b}\right)$.

(6) If $a>0$ and $a \neq 1$ and $b \geq 0$ and $c \geq 0$, then $b \cdot c \cdot \log _{a}(b \cdot c)=$ $b \cdot c \cdot \log _{a} b+b \cdot c \cdot \log _{a} c$.

(7) Let $q, q_{1}, q_{2}$ be finite sequences of elements of $\mathbb{R}$. Suppose len $q_{1}=\operatorname{len} q$ and len $q_{1}=\operatorname{len} q_{2}$ and for every $k$ such that $k \in \operatorname{dom} q_{1}$ holds $q(k)=$ $q_{1}(k)+q_{2}(k)$. Then $\sum q=\sum q_{1}+\sum q_{2}$.

(8) Let $q, q_{1}, q_{2}$ be finite sequences of elements of $\mathbb{R}$. Suppose len $q_{1}=\operatorname{len} q$ and len $q_{1}=\operatorname{len} q_{2}$ and for every $k$ such that $k \in \operatorname{dom} q_{1}$ holds $q(k)=$ $q_{1}(k)-q_{2}(k)$. Then $\sum q=\sum q_{1}-\sum q_{2}$.

(9) Suppose len $p \geq 1$. Then there exists $q$ such that len $q=\operatorname{len} p$ and $q(1)=$ $p(1)$ and for every $k$ such that $0 \neq k$ and $k<\operatorname{len} p$ holds $q(k+1)=$ $q(k)+p(k+1)$ and $\sum p=q(\operatorname{len} p)$.

Let us consider $p$. Let us observe that $p$ is non-negative if and only if:

(Def. 1) For every $i$ such that $i \in \operatorname{dom} p$ holds $p(i) \geq 0$.

Let us note that there exists a finite sequence of elements of $\mathbb{R}$ which is non-negative.

The following proposition is true

(10) If $p$ is non-negative and $r \geq 0$, then $r \cdot p$ is non-negative.

Let us consider $p, k$. We say that $p$ has only one value in $k$ if and only if:

(Def. 2) $\quad k \in \operatorname{dom} p$ and for every $i$ such that $i \in \operatorname{dom} p$ and $i \neq k$ holds $p(i)=0$.

Next we state four propositions:

(11) If $p$ has only one value in $k$ and $i \neq k$, then $p(i)=0$.

(12) If len $p=$ len $q$ and $p$ has only one value in $k$, then $p \bullet q$ has only one value in $k$ and $(p \bullet q)(k)=p(k) \cdot q(k)$.

(13) If $p$ has only one value in $k$, then $\sum p=p(k)$.

(14) If $p$ is non-negative, then for every $k$ such that $k \in \operatorname{dom} p$ and $p(k)=\sum p$ holds $p$ has only one value in $k$.

Let us observe that every finite sequence of elements of $\mathbb{R}$ which is finite probability distribution is also non empty and non-negative.

One can prove the following propositions:

(15) Let $p$ be finite probability distribution finite sequence of elements of $\mathbb{R}$ and given $k$ such that $k \in \operatorname{dom} p$ and $p(k)=1$. Then $p$ has only one value in $k$. 
(16) Let $i$ be a non empty natural number. Then $i \mapsto \frac{1}{i}$ is finite probability distribution finite sequence of elements of $\mathbb{R}$.

One can check that every matrix over $\mathbb{R}$ which is summable-to- 1 is also non empty yielding and every matrix over $\mathbb{R}$ which is joint probability is also non empty yielding.

The following propositions are true:

(17) For every matrix $M$ over $\mathbb{R}$ such that $M=\emptyset$ holds SumAll $M=0$.

(18) For every matrix $M$ over $D$ and for every $i$ such that $i \in \operatorname{dom} M$ holds $\operatorname{dom} M(i)=\operatorname{Seg}$ width $M$.

(19) $M_{1}$ is nonnegative iff for every $i$ such that $i \in \operatorname{dom} M_{1}$ holds $\operatorname{Line}\left(M_{1}, i\right)$ is non-negative.

\section{Properties of Transformations between Vector and Matrix}

Next we state four propositions:

(20) For every $j$ such that $j \in \operatorname{dom} p$ holds (LineVec2Mx $p)_{\square, j}=\langle p(j)\rangle$.

(21) Let $p$ be a non empty finite sequence of elements of $\mathbb{R}, q$ be a finite sequence of elements of $\mathbb{R}$, and $M$ be a matrix over $\mathbb{R}$. Then $M=$ ColVec2Mx $p \cdot \operatorname{LineVec} 2 \mathrm{Mx} q$ if and only if the following conditions are satisfied:

(i) $\quad$ len $M=\operatorname{len} p$,

(ii) width $M=$ len $q$, and

(iii) for all $i, j$ such that $\langle i, j\rangle \in$ the indices of $M$ holds $M_{i, j}=p(i) \cdot q(j)$.

(22) Let $p$ be a non empty finite sequence of elements of $\mathbb{R}, q$ be a finite sequence of elements of $\mathbb{R}$, and $M$ be a matrix over $\mathbb{R}$. Then $M=$ ColVec2Mx $p \cdot \operatorname{LineVec} 2 \mathrm{Mx} q$ if and only if the following conditions are satisfied:

(i) $\quad \operatorname{len} M=\operatorname{len} p$,

(ii) width $M=\operatorname{len} q$, and

(iii) for every $i$ such that $i \in \operatorname{dom} M \operatorname{holds} \operatorname{Line}(M, i)=p(i) \cdot q$.

(23) Let $p, q$ be finite probability distribution finite sequences of elements of $\mathbb{R}$. Then ColVec $2 \mathrm{Mx} p \cdot \operatorname{LineVec} 2 \mathrm{Mx} q$ is joint probability.

Let us consider $n$ and let $M_{1}$ be a matrix over $\mathbb{R}$ of dimension $n$. We say that $M_{1}$ is diagonal if and only if:

(Def. 3) For all $i, j$ such that $\langle i, j\rangle \in$ the indices of $M_{1}$ and $\left(M_{1}\right)_{i, j} \neq 0$ holds $i=j$.

Let us consider $n$. Observe that there exists a matrix over $\mathbb{R}$ of dimension $n$ which is diagonal.

The following proposition is true 
(24) Let $M_{1}$ be a matrix over $\mathbb{R}$ of dimension $n$. Then $M_{1}$ is diagonal if and only if for every $i$ such that $i \in \operatorname{dom} M_{1}$ holds Line $\left(M_{1}, i\right)$ has only one value in $i$.

Let us consider $p$. The functor Vec2DiagMx $p$ yielding a diagonal matrix over $\mathbb{R}$ of dimension len $p$ is defined as follows:

(Def. 4) For every $j$ such that $j \in \operatorname{dom} p$ holds $(\operatorname{Vec} 2 \operatorname{DiagMx} p)_{j, j}=p(j)$.

One can prove the following propositions:

(25) $\quad M_{1}=\operatorname{Vec} 2 \operatorname{DiagMx} p$ iff len $M_{1}=\operatorname{len} p$ and width $M_{1}=\operatorname{len} p$ and for every $i$ such that $i \in \operatorname{dom} M_{1}$ holds $\operatorname{Line}\left(M_{1}, i\right)$ has only one value in $i$ and Line $\left(M_{1}, i\right)(i)=p(i)$.

(26) Suppose len $p=$ len $M_{1}$. Then $M_{2}=\operatorname{Vec} 2 \operatorname{DiagMx} p \cdot M_{1}$ if and only if the following conditions are satisfied:

(i) $\quad$ len $M_{2}=$ len $p$,

(ii) width $M_{2}=$ width $M_{1}$, and

(iii) for all $i, j$ such that $\langle i, j\rangle \in$ the indices of $M_{2}$ holds $\left(M_{2}\right)_{i, j}=p(i)$. $\left(M_{1}\right)_{i, j}$.

(27) If len $p=\operatorname{len} M_{1}$, then $M_{2}=\operatorname{Vec} 2 \operatorname{DiagMx} p \cdot M_{1}$ iff len $M_{2}=\operatorname{len} p$ and width $M_{2}=$ width $M_{1}$ and for every $i$ such that $i \in \operatorname{dom} M_{2}$ holds $\operatorname{Line}\left(M_{2}, i\right)=p(i) \cdot \operatorname{Line}\left(M_{1}, i\right)$.

(28) Let $p$ be finite probability distribution finite sequence of elements of $\mathbb{R}$ and $M$ be a non empty yielding conditional probability matrix over $\mathbb{R}$. If len $p=$ len $M$, then Vec2DiagMx $p \cdot M$ is joint probability.

(29) Let $M$ be a matrix over $D$ and $p$ be a finite sequence of elements of $D^{*}$. Suppose len $p=$ len $M$ and $p(1)=M(1)$ and for every $k$ such that $k \geq 1$ and $k<$ len $M$ holds $p(k+1)=p(k)^{\frown} M(k+1)$. Let given $k$. If $k \in \operatorname{dom} p$, then len $p(k)=k \cdot$ width $M$.

(30) Let $M$ be a matrix over $D$ and $p$ be a finite sequence of elements of $D^{*}$. Suppose len $p=$ len $M$ and $p(1)=M(1)$ and for every $k$ such that $k \geq 1$ and $k<$ len $M$ holds $p(k+1)=p(k)^{\frown} M(k+1)$. Let given $i, j$. If $i \in \operatorname{dom} p$ and $j \in \operatorname{dom} p$ and $i \leq j$, then $\operatorname{dom} p(i) \subseteq \operatorname{dom} p(j)$.

(31) Let $M$ be a matrix over $D$ and $p$ be a finite sequence of elements of $D^{*}$. Suppose len $p=\operatorname{len} M$ and $p(1)=M(1)$ and for every $k$ such that $k \geq 1$ and $k<$ len $M$ holds $p(k+1)=p(k)^{\frown} M(k+1)$. Then len $p(1)=$ width $M$ and for every $j$ such that $\langle 1, j\rangle \in$ the indices of $M$ holds $j \in \operatorname{dom} p(1)$ and $p(1)(j)=M_{1, j}$.

(32) Let $M$ be a matrix over $D$ and $p$ be a finite sequence of elements of $D^{*}$. Suppose len $p=\operatorname{len} M$ and $p(1)=M(1)$ and for every $k$ such that $k \geq 1$ and $k<$ len $M$ holds $p(k+1)=p(k)^{\frown} M(k+1)$. Let given $j$. If $j \geq 1$ and $j<\operatorname{len} p$, then for every $l$ such that $l \in \operatorname{dom} p(j)$ holds $p(j)(l)=p(j+1)(l)$.

(33) Let $M$ be a matrix over $D$ and $p$ be a finite sequence of elements of $D^{*}$. 
Suppose len $p=$ len $M$ and $p(1)=M(1)$ and for every $k$ such that $k \geq 1$ and $k<$ len $M$ holds $p(k+1)=p(k)^{\frown} M(k+1)$. Let given $i, j$. Suppose $i \in \operatorname{dom} p$ and $j \in \operatorname{dom} p$ and $i \leq j$. Let given $l$. If $l \in \operatorname{dom} p(i)$, then $p(i)(l)=p(j)(l)$.

(34) Let $M$ be a matrix over $D$ and $p$ be a finite sequence of elements of $D^{*}$. Suppose len $p=$ len $M$ and $p(1)=M(1)$ and for every $k$ such that $k \geq 1$ and $k<$ len $M$ holds $p(k+1)=p(k)^{\frown} M(k+1)$. Let given $j$. Suppose $j \geq 1$ and $j<\operatorname{len} p$. Let given $l$. If $l \in \operatorname{Seg}$ width $M$, then $j \cdot$ width $M+l \in \operatorname{dom} p(j+1)$ and $p(j+1)(j \cdot$ width $M+l)=M(j+1)(l)$.

(35) Let $M$ be a matrix over $D$ and $p$ be a finite sequence of elements of $D^{*}$. Suppose len $p=$ len $M$ and $p(1)=M(1)$ and for every $k$ such that $k \geq 1$ and $k<$ len $M$ holds $p(k+1)=p(k)^{\frown} M(k+1)$. Let given $i, j$. Suppose $\langle i, j\rangle \in$ the indices of $M$. Then $(i-1) \cdot$ width $M+j \in \operatorname{dom} p(i)$ and $M_{i, j}=p(i)((i-1) \cdot$ width $M+j)$.

(36) Let $M$ be a matrix over $D$ and $p$ be a finite sequence of elements of $D^{*}$. Suppose len $p=\operatorname{len} M$ and $p(1)=M(1)$ and for every $k$ such that $k \geq 1$ and $k<$ len $M$ holds $p(k+1)=p(k)^{\frown} M(k+1)$. Let given $i, j$. Suppose $\langle i, j\rangle \in$ the indices of $M$. Then $(i-1) \cdot$ width $M+j \in \operatorname{dom} p(\operatorname{len} M)$ and $M_{i, j}=p(\operatorname{len} M)((i-1) \cdot$ width $M+j)$.

(37) Let $M$ be a matrix over $\mathbb{R}$ and $p$ be a finite sequence of elements of $\mathbb{R}^{*}$. Suppose len $p=\operatorname{len} M$ and $p(1)=M(1)$ and for every $k$ such that $k \geq 1$ and $k<$ len $M$ holds $p(k+1)=p(k)^{\frown} M(k+1)$. Let given $k$. If $k \geq 1$ and $k<$ len $M$, then $\sum p(k+1)=\sum p(k)+\sum M(k+1)$.

(38) Let $M$ be a matrix over $\mathbb{R}$ and $p$ be a finite sequence of elements of $\mathbb{R}^{*}$. Suppose len $p=\operatorname{len} M$ and $p(1)=M(1)$ and for every $k$ such that $k \geq 1$ and $k<$ len $M$ holds $p(k+1)=p(k)^{\frown} M(k+1)$. Then SumAll $M=$ $\sum p(\operatorname{len} M)$.

Let $D$ be a non empty set and let $M$ be a matrix over $D$. The functor Mx2FinS $M$ yields a finite sequence of elements of $D$ and is defined by:

(Def. 5)(i) $\quad \operatorname{Mx} 2$ FinS $M=\emptyset$ if len $M=0$,

(ii) there exists a finite sequence $p$ of elements of $D^{*}$ such that Mx2FinS $M=p(\operatorname{len} M)$ and len $p=\operatorname{len} M$ and $p(1)=M(1)$ and for every $k$ such that $k \geq 1$ and $k<$ len $M$ holds $p(k+1)=p(k)^{\frown} M(k+1)$, otherwise.

We now state several propositions:

(39) For every matrix $M$ over $D$ holds len Mx2FinS $M=\operatorname{len} M \cdot$ width $M$.

(40) Let $M$ be a matrix over $D$ and given $i, j$. If $\langle i, j\rangle \in$ the indices of $M$, then $(i-1) \cdot$ width $M+j \in \operatorname{dom} \operatorname{Mx} 2 \operatorname{FinS} M$ and $M_{i, j}=(\operatorname{Mx} 2 \operatorname{FinS} M)((i-$ 1) width $M+j)$.

(41) Let $M$ be a matrix over $D$ and given $k, l$. Suppose $k \in \operatorname{dom} \operatorname{Mx} 2$ FinS $M$ 
and $l=k-1$. Then $\langle(l \div$ width $M)+1,(l \bmod$ width $M)+1\rangle \in$ the indices of $M$ and $(\mathrm{Mx} 2 \mathrm{FinS} M)(k)=M_{(l \div \text { width } M)+1,(l \bmod \text { width } M)+1}$.

(42) SumAll $M_{1}=\sum \operatorname{Mx} 2 \operatorname{FinS} M_{1}$.

(43) $\quad M_{1}$ is nonnegative iff $\mathrm{Mx} 2 \mathrm{FinS} M_{1}$ is non-negative.

(44) $M_{1}$ is joint probability iff $\mathrm{Mx} 2 \mathrm{FinS} M_{1}$ is finite probability distribution.

(45) Let $p, q$ be finite probability distribution finite sequences of elements of $\mathbb{R}$. Then Mx2FinS(ColVec2Mx $p \cdot \operatorname{LineVec} 2 \mathrm{Mx} q)$ is finite probability distribution.

(46) Let $p$ be finite probability distribution finite sequence of elements of $\mathbb{R}$ and $M$ be a non empty yielding conditional probability matrix over $\mathbb{R}$. If len $p=\operatorname{len} M$, then $\operatorname{Mx} 2 \operatorname{FinS}(\operatorname{Vec} 2 \operatorname{DiagMx} p \cdot M)$ is finite probability distribution.

\section{INFORMATION ENTROPY}

Let us consider $a, p$. Let us assume that $a>0$ and $a \neq 1$ and $p$ is nonnegative. The functor $\overrightarrow{\log }_{a} p$ yields a finite sequence of elements of $\mathbb{R}$ and is defined by:

(Def. 6) len $\overrightarrow{\log }_{a} p=\operatorname{len} p$ and for every $k$ such that $k \in \operatorname{dom} \overrightarrow{\log }_{a} p$ holds if $p(k)>0$, then $\left(\overrightarrow{\log }_{a} p\right)(k)=\log _{a} p(k)$ and if $p(k)=0$, then $\left(\overrightarrow{\log }_{a} p\right)(k)=0$.

Let us consider $p$. The functor $\overrightarrow{\mathrm{id} \log p}$ yields a finite sequence of elements of $\mathbb{R}$ and is defined by:

(Def. 7) $\quad \overrightarrow{\mathrm{id} \log } p=p \bullet \overrightarrow{\log }_{2} p$.

The following propositions are true:

(47) Let $p$ be a non-negative finite sequence of elements of $\mathbb{R}$ and given $q$.

Then $q=\overrightarrow{\mathrm{id} \log } p$ if and only if the following conditions are satisfied:

(i) $\operatorname{len} q=\operatorname{len} p$, and

(ii) for every $k$ such that $k \in \operatorname{dom} q$ holds $q(k)=p(k) \cdot \log _{2} p(k)$.

(48) Let $p$ be a non-negative finite sequence of elements of $\mathbb{R}$ and given $k$ such that $k \in \operatorname{dom} p$. Then

(i) if $p(k)=0$, then $(\overrightarrow{\mathrm{id} \log } p)(k)=0$, and

(ii) if $p(k)>0$, then $(\overrightarrow{\mathrm{id} \log } p)(k)=p(k) \cdot \log _{2} p(k)$.

(49) Let $p$ be a non-negative finite sequence of elements of $\mathbb{R}$ and given $q$. Then $q=-\overrightarrow{\mathrm{id} \log } p$ if and only if the following conditions are satisfied:

(i) $\quad$ len $q=\operatorname{len} p$, and

(ii) for every $k$ such that $k \in \operatorname{dom} q$ holds $q(k)=p(k) \cdot \log _{2}\left(\frac{1}{p(k)}\right)$.

(50) Let $p$ be a non-negative finite sequence of elements of $\mathbb{R}$. Suppose $r_{1} \geq 0$ and $r_{2} \geq 0$. Let given $i$. If $i \in \operatorname{dom} p$ and $p(i)=r_{1} \cdot r_{2}$, then $(\overrightarrow{\text { id } \log p} p)(i)=$ $r_{1} \cdot r_{2} \cdot \log _{2} r_{1}+r_{1} \cdot r_{2} \cdot \log _{2} r_{2}$. 
(51) For every non-negative finite sequence $p$ of elements of $\mathbb{R}$ such that $r \geq 0$ holds $\overrightarrow{\mathrm{id} \log r} \cdot p=r \cdot \log _{2} r \cdot p+r \cdot\left(p \bullet \overrightarrow{\log }_{2} p\right)$.

(52) Let $p$ be a non empty finite probability distribution finite sequence of elements of $\mathbb{R}$ and given $k$. If $k \in \operatorname{dom} p$, then $(\overrightarrow{\mathrm{id} \log p})(k) \leq 0$.

Let us consider $M_{1}$. Let us assume that $M_{1}$ is nonnegative. The functor $\overrightarrow{\mathrm{id} \log } M_{1}$ yields a matrix over $\mathbb{R}$ and is defined as follows:

(Def. 8) len $\overrightarrow{\mathrm{id} \log } M_{1}=$ len $M_{1}$ and width $\overrightarrow{\mathrm{id} \log } M_{1}=$ width $M_{1}$ and for every $k$ such that $k \in \operatorname{dom} \overrightarrow{\mathrm{id} \log } M_{1}$ holds $\left(\overrightarrow{\mathrm{id} \log } M_{1}\right)(k)=\operatorname{Line}\left(M_{1}, k\right)$ $\overrightarrow{\log }_{2} \operatorname{Line}\left(M_{1}, k\right)$.

The following two propositions are true:

(53) For every nonnegative matrix $M$ over $\mathbb{R}$ and for every $k$ such that $k \in$ $\operatorname{dom} M$ holds Line $(\overrightarrow{\mathrm{id} \log } M, k)=\overrightarrow{\mathrm{id} \log } \operatorname{Line}(M, k)$.

(54) Let $M$ be a nonnegative matrix over $\mathbb{R}$ and $M_{3}$ be a matrix over $\mathbb{R}$. Then $M_{3}=\overrightarrow{\mathrm{id} \log } M$ if and only if the following conditions are satisfied:

(i) $\operatorname{len} M_{3}=$ len $M$,

(ii) width $M_{3}=$ width $M$, and

(iii) for all $i, j$ such that $\langle i, j\rangle \in$ the indices of $M_{3}$ holds $\left(M_{3}\right)_{i, j}=M_{i, j}$. $\log _{2}\left(M_{i, j}\right)$.

Let $p$ be a finite sequence of elements of $\mathbb{R}$. The functor Entropy $p$ yields a real number and is defined by:

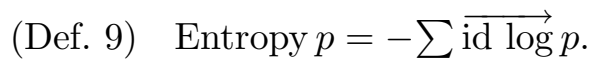

We now state several propositions:

(55) For every non empty finite probability distribution finite sequence $p$ of elements of $\mathbb{R}$ holds Entropy $p \geq 0$.

(56) Let $p$ be a non empty finite probability distribution finite sequence of elements of $\mathbb{R}$. If there exists $k$ such that $k \in \operatorname{dom} p$ and $p(k)=1$, then Entropy $p=0$.

(57) Let $p, q$ be non empty finite probability distribution finite sequences of elements of $\mathbb{R}$ and $p_{1}, q_{3}$ be finite sequences of elements of $\mathbb{R}$. Suppose that

(i) $\operatorname{len} p=\operatorname{len} q$,

(ii) $\operatorname{len} p_{1}=\operatorname{len} p$

(iii) $\quad$ len $q_{3}=\operatorname{len} q$, and

(iv) for every $k$ such that $k \in \operatorname{dom} p$ holds $p(k)>0$ and $q(k)>0$ and $p_{1}(k)=-p(k) \cdot \log _{2} p(k)$ and $q_{3}(k)=-p(k) \cdot \log _{2} q(k)$.

Then

(v) $\sum p_{1} \leq \sum q_{3}$

(vi) for every $k$ such that $k \in \operatorname{dom} p$ holds $p(k)=q(k)$ iff $\sum p_{1}=\sum q_{3}$, and

(vii) there exists $k$ such that $k \in \operatorname{dom} p$ and $p(k) \neq q(k)$ iff $\sum p_{1}<\sum q_{3}$. 
(58) Let $p$ be a non empty finite probability distribution finite sequence of elements of $\mathbb{R}$. Suppose that for every $k$ such that $k \in \operatorname{dom} p$ holds $p(k)>$ 0 . Then

(i) Entropy $p \leq \log _{2}$ len $p$,

(ii) for every $k$ such that $k \in \operatorname{dom} p$ holds $p(k)=\frac{1}{\operatorname{len} p}$ iff Entropy $p=$ $\log _{2} \operatorname{len} p$, and

(iii) there exists $k$ such that $k \in \operatorname{dom} p$ and $p(k) \neq \frac{1}{\operatorname{len} p}$ iff Entropy $p<$ $\log _{2} \operatorname{len} p$.

(59) For every nonnegative matrix $M$ over $\mathbb{R}$ holds Mx2FinS $\overrightarrow{\text { id } \log } M=$ $\underset{\text { id } \log }{\longrightarrow}$ Mx2FinS $M$.

(60) Let $p, q$ be finite probability distribution finite sequences of elements of $\mathbb{R}$ and $M$ be a matrix over $\mathbb{R}$. If $M=\operatorname{ColVec} 2 \mathrm{Mx} p \cdot \operatorname{LineVec} 2 \mathrm{Mx} q$, then SumAll $\overrightarrow{\mathrm{id} \log } M=\sum \overrightarrow{\mathrm{id} \log } p+\sum \overrightarrow{\mathrm{id} \log } q$.

Let us consider $M_{1}$. The entropy of joint probability of $M_{1}$ yields a real number and is defined as follows:

(Def. 10) The entropy of joint probability of $M_{1}=$ Entropy Mx2FinS $M_{1}$.

Next we state the proposition

(61) Let $p, q$ be finite probability distribution finite sequences of elements of $\mathbb{R}$. Then the entropy of joint probability of ColVec $2 \mathrm{Mx} p \cdot \operatorname{LineVec} 2 \mathrm{Mx} q=$ Entropy $p+$ Entropy $q$.

Let us consider $M_{1}$. The entropy of conditional probability of $M_{1}$ yields a finite sequence of elements of $\mathbb{R}$ and is defined by the conditions (Def. 11).

(Def. 11)(i) len (the entropy of conditional probability of $M_{1}$ ) $=\operatorname{len} M_{1}$, and

(ii) for every $k$ such that $k \in \operatorname{dom}$ (the entropy of conditional probability of $M_{1}$ ) holds (the entropy of conditional probability of $\left.M_{1}\right)(k)=$ Entropy Line $\left(M_{1}, k\right)$.

One can prove the following propositions:

(62) Let $M$ be a non empty yielding conditional probability matrix over $\mathbb{R}$ and $p$ be a finite sequence of elements of $\mathbb{R}$. Then $p=$ the entropy of conditional probability of $M$ if and only if len $p=\operatorname{len} M$ and for every $k$ such that $k \in \operatorname{dom} p$ holds $p(k)=-\sum(\overrightarrow{\mathrm{id} \log } M)(k)$.

(63) Let $M$ be a non empty yielding conditional probability matrix over $\mathbb{R}$. Then the entropy of conditional probability of $M=-$ LineSum id $\overrightarrow{\log } M$.

(64) Let $p$ be finite probability distribution finite sequence of elements of $\mathbb{R}$ and $M$ be a non empty yielding conditional probability matrix over $\mathbb{R}$. Suppose len $p=\operatorname{len} M$. Let $M_{3}$ be a matrix over $\mathbb{R}$. If $M_{3}=\operatorname{Vec} 2 \operatorname{DiagMx} p \cdot M$, then SumAll $\overrightarrow{\mathrm{id} \log } M_{3}=\sum \overrightarrow{\mathrm{id} \log } p+\sum(p \bullet$ LineSum id $\log M$ ).

(65) Let $p$ be finite probability distribution finite sequence of elements of $\mathbb{R}$ and $M$ be a non empty yielding conditional probability matrix over 
$\mathbb{R}$. Suppose len $p=$ len $M$. Then the entropy of joint probability of Vec2DiagMx $p \cdot M=$ Entropy $p+\sum(p \bullet$ the entropy of conditional probability of $M)$.

\section{REFERENCES}

[1] Grzegorz Bancerek. The ordinal numbers. Formalized Mathematics, 1(1):91-96, 1990.

[2] Grzegorz Bancerek and Krzysztof Hryniewiecki. Segments of natural numbers and finite sequences. Formalized Mathematics, 1(1):107-114, 1990.

[3] P. Billingsley. Ergodic Theory and Information. John Wiley \& Sons, 1964.

[4] Czesław Byliński. Binary operations. Formalized Mathematics, 1(1):175-180, 1990.

[5] Czesław Byliński. Finite sequences and tuples of elements of a non-empty sets. Formalized Mathematics, 1(3):529-536, 1990.

[6] Czesław Byliński. Functions and their basic properties. Formalized Mathematics, 1(1):5565, 1990.

[7] Czesław Byliński. Functions from a set to a set. Formalized Mathematics, 1(1):153-164, 1990.

[8] Czesław Byliński. Some basic properties of sets. Formalized Mathematics, 1(1):47-53, 1990.

[9] Czesław Byliński. The sum and product of finite sequences of real numbers. Formalized Mathematics, 1(4):661-668, 1990.

[10] Agata Darmochwat. The Euclidean space. Formalized Mathematics, 2(4):599-603, 1991.

[11] Shigeichi Hirasawa. Information Theory. Baifukan CO., 1996.

[12] Katarzyna Jankowska. Matrices. Abelian group of matrices. Formalized Mathematics, 2(4):475-480, 1991.

[13] Artur Korniłowicz. On the real valued functions. Formalized Mathematics, 13(1):181-187, 2005.

[14] Jarosław Kotowicz. Real sequences and basic operations on them. Formalized Mathematics, 1(2):269-272, 1990.

[15] Rafał Kwiatek. Factorial and Newton coefficients. Formalized Mathematics, 1(5):887-890, 1990.

[16] Yatsuka Nakamura, Nobuyuki Tamaura, and Wenpai Chang. A theory of matrices of real elements. Formalized Mathematics, 14(1):21-28, 2006.

[17] Library Committee of the Association of Mizar Users. Binary operations on numbers. To appear in Formalized Mathematics.

[18] Konrad Raczkowski and Andrzej Nȩdzusiak. Real exponents and logarithms. Formalized Mathematics, 2(2):213-216, 1991.

[19] Yasunari Shidama. The Taylor expansions. Formalized Mathematics, 12(2):195-200, 2004.

[20] Andrzej Trybulec. Subsets of complex numbers. To appear in Formalized Mathematics.

[21] Andrzej Trybulec. Tarski Grothendieck set theory. Formalized Mathematics, 1(1):9-11, 1990.

[22] Wojciech A. Trybulec. Vectors in real linear space. Formalized Mathematics, 1(2):291$296,1990$.

[23] Zinaida Trybulec. Properties of subsets. Formalized Mathematics, 1(1):67-71, 1990.

[24] Edmund Woronowicz. Relations and their basic properties. Formalized Mathematics, 1(1):73-83, 1990.

[25] Bo Zhang and Yatsuka Nakamura. The definition of finite sequences and matrices of probability, and addition of matrices of real elements. Formalized Mathematics, 14(3):101108,2006 . 